\title{
Gravitational Energy Level and the Nature of Microwave Background of Universe
}

\author{
Vladimir Konushko \\ Protvino, Moscow region \\ E-mail: KONUSHKO@mail.ru \\ Received February 21, 2010; revised April 27, 2010; accepted May 23, 2010
}

\begin{abstract}
In 1965, Penzias and Wilson discovered thermal radiation with $T_{0} \sim 2.7 \mathrm{~K}$ further on called "relict". This article is concerned with the new phenomenon, i.e. the formation of gravitational energy levels by any body, with the result that photons are produced whose spectrum close to the Earth is similar to that of a blackbody with $T_{0} \sim 2.7 \mathrm{~K}$. The critical analysis of the experiments performed with the cosmic observatories COBE and WMAP completely confirms this prediction.
\end{abstract}

Keywords: Microwave Background, Relict

\section{Introduction}

In 1965 Penzias and Wilson reported that they had registered weak noise with a sensitive radiometer [1]. These signals were observed at the wavelength of $7.35 \mathrm{~cm}$. Later on precision radiometers were used to measure the radio signals at other frequencies. At present the absolute majority of physicists believe that this noise is caused by a thermal radiation corresponding to that of a blackbody with $T_{0}=(2.725 \pm 0.002) \mathrm{K}$ [2].

Nowadays the expansion of the Universe is considered working hypothesis but it is still just a hypothesis. Besides, it is assumed that at the beginning of the expansion the matter was hot (the Big bang hypothesis). The idea of a high temperature at the beginning of the expansion was put forward by G. Gamov in the mid-40 s. He also pointed out that, according to his hypothesis, today's Universe contains relict radiation cooled by expansion. In collaboration with R.Alfer he evaluated its approximate temperature, $T_{0} \sim 5 \mathrm{~K}$ [3].

But the following facts cast a shadow on this conclusion.

1) The defenders of the theory of Universe cooling on expansion ignore the results of Joule'sx experiment on free expansion of gas into vacuum referred to by K.Huang in his paper [4]. The experiment shows that, as an ideal gas expands into vacuum, its temperature remains constant, $T_{2}=T_{1}$.

2) Since the Universe is a closed system, the natural question arises: where does the huge energy caused by
Universe cooling disappear?

3) Thermal radiation only occurs when an equilibrium between the radiation and the substance takes place. As a result of the Big bang, all the elementary particles fly apart at nearly velocities of light, therefore the existence of an equilibrium state is most problematic for a bodyradiation system.

4) It should be stressed that there are several independent methods of deducing the Planck radiation formula for blackbody radiation spectrum, and a necessary requirement for it is discreteness of the spectrum of the photons formed as the electrons pass onto a low-lying level. The photons formed by the Big bang were at the state of complete chaos and discreteness was out of question at that time since there were no atoms at all. The above-said facts make us revise completely the physical nature of the cosmic microwave background (CMB).

\section{Energy Levels}

The investigations of I. Fraunhofer into the radiation of the solar spectrum and then spectra of terrestrial light sources were the beginning of spectroscopy. Analysis of experimental data enabled N. Bohr in 1913 to make a suggestion on the existence of discrete energy levels of atom completely supported by his numerous experiments. This study of Bohr is one of the most amazing phenomena in the history of science. The birth of this theory, before the wave properties of particles were cleared up, 
can be only explained by his genius. It was just on this point that Einstein said "... the highest musicality in the field of theoretical thought”.

Energy levels of atom arise due to Coulomb interaction of electrically charged particles, ultimately owing to the electric field. The magnetic field of atom contributes to the formation of energy levels, too: owing to spinorbit and spin-spin interactions additional levels arise near the ground levels. Such an extravagant phenomenon as the Lamb-Riserford shift cannot be ruled out from energy levels.

The development of nuclear physics has shown that nuclear interaction is characterized by the formation of nuclear energy levels as well.

It has been believed for a long time that the tensors entering into the Einstein equations are deformation and elasticity tensors of space structure. In this connection it is rather surprising that nobody studied gravitational energy levels and tried to discover them experimentally before 1975. The more surprising thing is that the Coulomb's and Newton's laws are very much alike, like twin brothers.

It was in 1975 that the author of this article proposed that any bunch of substance-from an elementary particle to an accumulation of galaxies-gives rise to gravitational energy levels around itself.

According to quantum mechanics, a potential-bound system, such as an oscillator, has a discrete set of energy levels. When studying gravitation we, however, make a more important statement: every isolated body forms its gravitational energy levels. This dissimilarity from quantum mechanics is related to the large difference between Ampere's and H.Oersted's discoveries.

\section{Gravitational Energy Levels of Earth and Sun}

The Schrödinger equation for the simplest hydrogen atom gives the arrangement of energy levels and permissible orbit radii

$$
r_{n}=\frac{\hbar}{m_{e} c} \cdot \frac{\hbar c}{e^{2}} \cdot n^{2}(n=1,2,3, \cdots) .
$$

The radius of the first orbit of hydrogen atom is expressed as

$$
r_{0}=\frac{\hbar}{m_{e} c} \cdot \frac{\hbar c}{e^{2}}=0.529 \times 10^{-8} \mathrm{~cm} .
$$

As far as gravitation is concerned, the question arises: what can be taken for $r_{0}$, when the Earth is considered to be the nucleus of a gravitational "atom"? First of all we should recall the unique property of spherical bodies: the gravitational force of the Earth at a point on its surface or above it is identical to the one in the case as though the whole mass of the Earth were concentrated in its centre. The size of the planet cannot enter into $r_{0}$ since its density could, in principle, vary over wide limits and the gravitational field would remain the same. Thus, this quantity should only include such world constant as $\hbar, G$, $c$ and the mass of the planet $M$.

The gravitational radius $r_{g}$ is both fundamental and puzzling for macroscopic bodies, for the Earth it equals

$$
r_{g}=\frac{2 G M_{\oplus}}{c^{2}} \approx 0.9 \mathrm{~cm} .
$$

Only this value can be taken for $r_{0}$ :

$$
r_{0}=r_{g}
$$

By analogy with quantum mechanics dealing with Coulombian fields, the radii of gravitational energy levels will be:

$$
r_{n}=r_{g} \cdot n^{2}=\frac{2 G M_{\oplus}}{c^{2}} \cdot n^{2} .
$$

The mean radius of the Earth $R_{0} \approx 6371 \mathrm{~km}$. In this case the quantum number $n$ for the energy levels near its surface

$$
n_{\oplus}=\sqrt{\frac{R_{\oplus}}{r_{g \oplus}}}=2.68 \times 10^{4}
$$

The eigenvalues of energy for a particle, with its mass $m$, at a distance $R$ larger than the size $R_{b}$ of $M, R>R_{b}$.

$$
\begin{aligned}
& E_{n}=\frac{G M}{r_{g}} \cdot \frac{1}{n^{2}} ;\left(n=n_{0}, n_{0}+1, \cdots\right) \\
& n_{0}=\sqrt{\frac{R_{b}}{r_{g}}}
\end{aligned}
$$

The emitted light frequency, as a gravitational "atom" passes from the state $n$ to the state $k$ is

$$
\omega=\frac{G M m}{r_{g} \hbar} \cdot\left(\frac{1}{k^{2}}-\frac{1}{n^{2}}\right) .
$$

Then the emitted photon energy can respectively be expressed as

$$
E_{\gamma}=\frac{G M m}{r_{g}} \cdot\left(\frac{1}{k^{2}}-\frac{1}{n^{2}}\right) .
$$

This formula is closely resembles the Balmer formula stressing again the mysterious relation between gravity and electricity. But, unlike the Balmer formula, Formula (6) covers a wide radiation spectrum, up to the energy levels of an accumulation of galaxies.

A simple way of checking our considerations is to calculate the velocity of any body on the nearest orbit of the Earth, that is, the circular orbital velocity $v_{1}$ 


$$
v_{1}=\frac{c}{\sqrt{2} \cdot n_{\oplus}} \approx 8 \times 10^{3} \mathrm{~m} / \mathrm{sec},
$$

where $c$ is the velocity at the first gravitational level $n=$ 1 , when $r=r_{g}$, which means that $c$ is the velocity of light and we act by analogy with quantum mechanics. Thus, the number $n_{\oplus}=2.68 \times 10^{4}$ is real and it confirms the correctness of the choice $r_{0}=r_{g}$.

The distance between two adjacent energy levels

$$
\Delta r=r_{n+1}-r_{n}=(2 n-1) \cdot r_{0}
$$

Near the Earth's surface it equals

$$
\Delta r_{\oplus} \approx 477 \mathrm{~m}
$$

This value, which is small as compared to the radius of the Earth, shows that the energy levels of such a gravitational "atom" near the planet are so densely arranged that, as electrons and protons pass onto a lower level the quanta emitted form a continuous radiation spectrum. It should be noted that the situation in atoms is quite different: the levels near the nucleus are very far-scattered. But what kind of quanta are they? At first sight, as we investigate gravity, they seem to be quanta of a gravitational field, that is, gravitons.

And, nevertheless, electrons and protons are most likely to emit photons and that is why. Any particle moving at a high speed cannot "remember" which physical field-electronic, nonuniform magnetic or gravitationalhas transmitted energy to it. Hence, any energy levels account for the deformation of space structure.

Leaving aside the very important problem of gravitons for awhile, we shall go on studying the spectra of photons only emitted by the electrons passing from one gravitational level of a macroscopic body (Earth, Sun, stars) onto another one.

The quantum number $n$ near the Sun's surface

$$
n_{\Theta}=\sqrt{\frac{R_{\Theta}}{r_{g}}}=483 .
$$

In this case the distance between adjacent energy levels nearly the Sun is

$$
\Delta r_{\Theta}=2900 \mathrm{~km} \text {. }
$$

This distance is extremely small, too, as compared to the size of the Sun and, hence, its gravitational radiation spectrum will be nearly continuous, too.

The basic decoration of the Saturn is its rings extending to about $60000 \mathrm{~km}$ from the planet Figure 1.

The flights of space vehicles have shown that the rings of the Saturn are not solid but consist of hundreds and thousands of individual rings one inside the other and separated by small "holes" of about $10 \mathrm{~km}$. It is rather interesting to calculate the distance between the gravita-

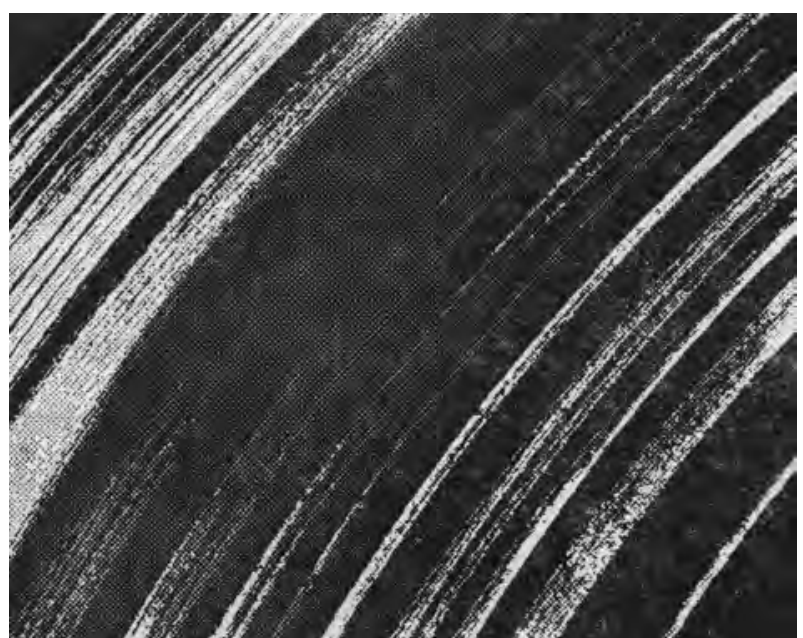

Figure 1. The fine structure of Saturn's rings.

tional energy levels of this wonderful planet near its surface using Formula (7) $\Delta r \approx 14 \mathrm{~km}$.

No doubt, when observing the fine structure of the Saturn's rings we can observe by the naked eye individual gravitational energy levels.

Later ringed systems of small particles and bodies were discovered around the Jupiter and the Uranus. These systems resist usual observations from the Earth but they have rather a complex structure.

Another surprising ability demonstrated by the Sun is that the distance between the adjacent gravitational levels formed by the Sun near the Earth's surface will be

$$
\Delta r=42100 \mathrm{~km}
$$

This number astonishingly coincides with the distance at which a geostationary sate like hanging over a certain point of the Earth must revolve

$$
R_{\oplus}+H=\sqrt[3]{\frac{G \cdot M \cdot T^{2}}{4 \pi^{2}}} \approx 42100 \mathrm{~km},
$$

where $T$ is the period of revolution of the planet, $H$ is the height over the Earth's surface.

There is no question that the Earth is on a gravitational level formed by the Sun. The short distances between adjacent energy levels near the surfaces of all the planets in the solar system indicate that they are all the positioned on energy levels. Hence we can make a very important conclusion: just as electrons in atoms are grouped on atomic energy levels, so cosmic dust and fragments are grouped on the gravitational levels set up by stars thus forming planets.

\section{Gravitational Quasi-Blackbodied Radiation}

In the above parts we adduced convincing arguments in 
favor of gravitational energy levels and now are going to consider the sequences of this wonderful prediction. The mean energy of black-bodied radiation with its frequency $\omega$ can be expressed as

$$
\langle\varepsilon\rangle=\frac{\hbar \omega}{\exp (\hbar \omega / k T)-1}
$$

Note that, with $\hbar$ tending to zero, Formula (8) changes to the classical expression

$$
\langle\varepsilon\rangle=k T
$$

This can be supported by setting $\exp (\hbar \omega / k T) \approx 1+$ $\hbar \omega / k T$, which can be met the more precisely, the less $\hbar$. Thus, if the energy could assume a continuous series of values, its mean value would be equal to $k T$.

As it has already been mentioned above, the gravitational energy levels near both the Earth and the Sun and, in general, nearby any body are arranged so densely that the radiation spectrum produced by electrons, as they pass from one gravitational level to another one, can be considered continuous quasi-blackbodied.

As for the atomic structure, the radiation for far removed orbits (that is, orbits more characterized by macroscopic conditions) found by the use of the Bohr theory as well as within the frameworks of quantum mechanics approximates to the radiation found by the Maxwell theory, that is, classical. For macroscopic bodies the quantum number $n$ is always large even near the surface (for the Earth $n=26$ 800) thus complying with the classical requirements. In the hydrogen atom the ground state is characterized by the quantum number $n=1$. That is why we have $k T$ in the right member of (9) but not $3 / 2 k T$.

It is known from thermodynamics that if a gas is in equilibrium state (that is, in a state with constant parameters), the velocity distribution remains unchanged. The electrons filling atoms the whole magnetosphere of the Earth meet this requirement in a first approximation, and one of the characteristics of this electron gas is the mean kinetic energy of electrons hitting the Earth.

From atomic physics it is evident that in excited atoms the electrons on the higher energy levels tend to go back to the nucleus if there are vacancies on the level $n=1$.

In a similar manner, in the gravitational "atom" where the nucleus is the Earth, the electrons at a large distance from the Earth try to pass under gravity onto the level closest to the planet and characterized by the circular orbital velocity $v_{1}$. There can be no doubt that it is just this velocity $v_{1}$ that is the most probable, $v_{p r}=v_{1}$.

The energy conservation law for an electron on a near-earth orbit takes the form:

$$
\frac{G M_{\oplus} m_{e}}{R_{\oplus}}=\frac{m_{e} v_{1}^{2}}{2},
$$

hence,

$$
v_{1}^{2}=\frac{G M_{\oplus}}{R_{\oplus}}
$$

The escape velocity $v_{2}$ is

$$
v_{2}^{2}=\frac{2 G M_{\oplus}}{R_{\oplus}}
$$

When the elementary gas theory is applied to electrons, the most probable velocity can be expressed as

$$
v_{p r}^{2}=\frac{2 k T}{m_{e}}
$$

On the assumption $v_{1}=v_{p r}$ we have

$$
v_{1}^{2}=\frac{2 k T}{m_{e}}
$$

The mean-square velocity of the electrons producing electron gas will be:

$$
\langle v\rangle^{2}=\frac{8 k T}{\pi m_{e}}
$$

The relation between $v_{1}^{2}$ and $\langle v\rangle^{2}$ becomes obvious

$$
\langle v\rangle^{2}=\frac{4 v_{1}^{2}}{\pi}
$$

The mean kinetic energy takes the form

$$
\langle\varepsilon\rangle=\frac{m\langle v\rangle^{2}}{2}=\frac{2 m_{e} v_{1}^{2}}{\pi}=\frac{3 G M_{\oplus} m_{e}}{\pi R_{\oplus}}
$$

Using Formula (9) we get

$$
\frac{2 G M_{\oplus} m_{e}}{\pi R_{\oplus}}=k T
$$

And, finally, the electron gas temperature near the Earth can be found

$$
T=\frac{2 G M_{\oplus} m_{e}}{\pi k R_{\oplus}} \approx 2.64 \mathrm{~K}
$$

Since the spectrum of emitted photons faithfully copies the spectrum of electrons, the above temperature can be considered the temperature of quasi-black-bodied radiation of the Earth.

This temperature value is surprisingly close to that of CMB measured by the Cosmic Background Explorer (COBE) [2].

$$
T_{0}=2.725 \pm 0.002 \mathrm{~K} \text {. }
$$

The whole ensuing material in this article will be sufficient proof of the fact that the coincidence of these temperatures is not accidental. 
Let us give some more calculations to show the validity of the result obtained and exclude the probability of errors. If we make all the calculations replacing the mean velocity by the circular orbital velocity $v_{1}$, the radiation temperature will be

$$
T_{1} \approx 2.07 \mathrm{~K} \text {. }
$$

The maximum (the escape velocity $v_{2}$ ) velocity will enable us to find

$$
T_{2} \approx 4.14 \mathrm{~K} \text {. }
$$

Thus, even the ultimate temperatures of quasi-blackbodied radiation of the Earth are close to that of CMB $T_{0}$ which rules out the probability of on error. Attention must be given to the following two moments:

1) In our considerations we did not use any matching parameters.

2) The emitted photon spectrum is strictly discrete, which is the basic requirement for deducing the Planck formula.

\section{Gravitational Energy Levels of the Sun}

The consideration of the temperature on the Sun's surface gives another argument in favor of the existence of gravitational energy levels. As it has been mentioned, the radiation spectrum formed by the gravitational levels of the Sun can be considered quasi-blackbodied. It should be noted, however, that the high temperature of the Sun does not allow most electrons to reach the sun's surface, so we must substitute $v_{1}^{2}$ in Formula (11) for $\langle v\rangle^{2}$

$$
\frac{G M_{\Theta} m_{e}}{2 R_{\Theta}}=k T_{\Theta},
$$

hence:

$$
T_{\Theta}=6285 \mathrm{~K} .
$$

The maximum solar radiation falls on $\lambda_{\max } \approx 4.6 \times$ $10^{-5} \mathrm{~cm}$. The temperature calculated by the Wien displacement law

$$
T_{\Theta} \approx 6300 \mathrm{~K} .
$$

The accurate coincidence of both the temperatures is another decisive evidence of the existence of gravitational energy levels near any body.

The effective surface temperature of the Sun $T_{e f}=$ $5785 \mathrm{~K}$, which is less than the theoretical value.

This difference is usually explained by the fact that the solar spectrum is not exactly black-bodied which is due to the existence of a stellar boundary where the thermodynamic equilibrium condition is disturbed.

The true cause is that not all electrons reach the solar surface passing from one gravitational level onto another one (owing to a high temperature) and some of them are taken away by the solar wind that feeds the Earth's surface with electrons. Therefore the spectrum maximum remains constant while the intensity drops thus decreasing the solar temperature.

The surface temperatures of stationary stars, usually in the Herzsprung-Ressel sequence, are dictated by gravitational levels, too. Simple calculations show that, when a neutron star is born and some time after, its temperature may be as high as nearly $10^{9} \mathrm{~K}$ and again owing to gravitational levels.

Let us next consider an interesting physical phenomenon characteristic of both the Earth and the Sun and which has not a satisfactory numerical explanation. The average temperature if the Earth's underlying surface is $\sim 15^{\circ} \mathrm{C}$. The photosphere reaches $50 \mathrm{~km}$. Its specific feature is that the temperature increases with height due to the formation of ozone $\left(\mathrm{O}_{3}\right)$. At a height of $55 \mathrm{~km}$ the temperature increases up to $0^{\circ} \mathrm{C}$. Above $55 \mathrm{~km}$ and up to $80 \mathrm{~km}$ it drops to $-85^{\circ} \mathrm{C}$. The thermosphere begins above $100 \mathrm{~km}$, the temperature here increases drastically and at $400 \mathrm{~km}$ it may be as high as $\sim 1200^{\circ} \mathrm{C}$. The exosphere above the thermosphere forms its outer shell. The temperature in the exosphere is high too, and this is a mystery of nature. What heats the extremely rarefied atmosphere?

This effect is normally explained by the fact that the temperature rises because the terrestrial atmosphere absorbs the UV radiation of the Sun.

Gravitational levels "prompt" us a more interesting physical phenomenon. We have so for considered just the transition of electrons from one gravitational level onto another one and their attendant photon emission, but the inner van Allen radiation belt contains a certain number of protons. Since the mass of a proton is 1836 times larger than that of an electron, the transition of protons onto lower energy levels of the Earth will form its own spectrum with $T \sim 5000^{\circ} \mathrm{C}$. This would occur, however, in an ideal case if there were a sufficient quantity of protons and they all could reach the Earth's surface. Actually this is not the case, and only the upper layers of the atmosphere are heated to a much lower temperature $\sim 1200^{\circ} \mathrm{C}$.

This situation is almost similar to the solar atmosphere, where the thickest layer $\sim 300 \mathrm{~km}$, is called the photosphere. In this layer the temperature decreases farther and farther away from the centre varying between 8000 and $4000 \mathrm{~K}$. In the next layer, the chromospheres, it rapidly rises reaching hundreds of thousands of K. Above the chromospheres the solar gas temperature may be as high as $\sim 2 \times 10^{6} \mathrm{~K}$ and father, over a length of many solar radii, it does not practically change!

Some new information on solar atmosphere was obtained in September of 2006 when a space Hinode vehi- 
cle was launched. This information corroborates fully the abnormally high temperature of the solar corona-over $2000000 \mathrm{~K}$.

The heating of the upper layers of the solar atmosphere is traditionally believed to be caused by the wave motion of substance arising in the convectional zone. These waves pass through the photosphere and carry into the chromospheres and the corona a small fraction of the mechanical energy which the gases in the convectional zone possess.

But the outer surface of the photosphere has a temperature just $\sim 4000 \mathrm{~K}$ and the above explanation does not stand up.

Let's turn our attention to the gravitational levels of the Sun again. The temperature, which would be set up as a result of proton emission on the energy levels on the solar surface, would reach $\sim 11 \times 10^{6} \mathrm{~K}$. This value is nearly equal to the temperature of the solar core where thermonuclear reactions proceed.

We shall have a more striking result if we consider the initial period when the incipient Sun can be considered cold and we have to use $a\langle v\rangle^{2}$ instead of $v_{1}^{2}$ in temperature calculations.

$$
\langle v\rangle^{2}=\frac{8}{2 \pi} v_{1}^{2} .
$$

The solar surface temperature in this case may be as high as $14 \times 10^{6} \mathrm{~K}$ (when thermonuclear reactions start). Consequently, gravitational energy levels can be a match that lights stars.

And again the deficient number of free protons (a part of them fly away into word space in the form of a solar wind) and the high solar surface temperature, at which highly excited atoms can emit, allow the effect under consideration to heat just the upper layers of the solar atmosphere up to $2 \times 10^{6} \mathrm{~K}$.

\section{The Fine Structure of the Earth's Gravitational Potential and Its Consequences}

The temperature ( $T \approx 2.64 \mathrm{~K}$ ) of the microwave background set up by the Earth has been obtained by us assuming that the planet is shaped like an ideal ball with its radius $R_{\oplus} \approx 6371 \mathrm{~km}$.

But the Earth's surface is described in fact by an individual figure called a geoid [5].

The difference between the surface of a geoid and an ellipsoid (spheroid) does not exceed several tens of meters, whereas the difference between the equatorial and the polar radii $\left(R_{e}\right.$ and $\left.R_{p}\right)$ comes to $21.385 \mathrm{~km}$. The geoid oblateness a equal to

$$
a=\frac{R_{e}-R_{p}}{R_{e}}=\frac{1}{298.255} \approx 0.0033 .
$$

According to Newton's law, the attraction of a unit mass by an element of mass $d m$ at a distance $r$ can be expressed as

$$
F=\frac{G d m}{r^{2}},
$$

where $G$ is the gravitational constant. The potential of attraction by a body at a point out side it in this case will be

$$
U=G \int \frac{\mathrm{d} m}{r} .
$$

The solution of the equation presents an infinite series the coefficients of which are Legendre polynomials $P_{n}(\cos \Theta)$, which arise in our consideration in a natural way. The first correction term for Equation (13) was determined even before launching artificial Earth satellites by means of ground measurements [5].

$$
U=\frac{G M_{\oplus}}{R}\left[1-\left(\frac{R_{e}}{R}\right) \cdot I_{2} \cdot P_{2}(\cos \theta)\right],
$$

where $I_{2}$ is the quadrupole amplitude the analogue of which in the WMAP experiment [2] is the CMB anisotropy amplitude $\mathrm{C}_{1}$.

The value of $I_{2}$ is equal to $1082.65 \times 10^{-6}$ which causes the quadrupole expansion term to contribute to the CMB temperature

$$
\Delta T_{2}=T \cdot \Delta U \approx I_{2} \cdot T \approx 3 \mathrm{mK} .
$$

This value remarkably coincides with that of the dipole amplitude $l=1$ measured by the COBE [2]

$$
\Delta T_{1}=(3.353 \pm 0.024) \mathrm{mK}
$$

This result was adjusted by the WMAP

$$
\Delta T_{1}=(3.346 \pm 0.017) \mathrm{mK}
$$

A very important fact for us is that the WMAP did not directly measure the dipole but obtained this value by determining the residual dipole in processing.

The quadrupole $l=2$ measured by the WMAP has a very low amplitude

$$
\Delta T_{2}^{2}=(154 \pm 70) \mu \mathrm{K}^{2}
$$

or

$$
\Delta T_{2}=(12.5 \pm 8.5) \mu \mathrm{K} .
$$

To understand the situation involving the quadrupole we should point up to the following.

The multipole expansion of the gravitational potential allowed finding the contribution of the quadrupole $\approx 3$ 
mK to the temperature $T_{0}$ of microwave background. The WMAP, however, determined the anisotropy of quadrupole rather then the temperature or the gradient of quadrupole. We should stress again that the difference in height between a genoid and a spheroid does not exceed $a^{2} \times R \approx 70 \mathrm{~m}$ which makes up a negligible quantity $1.1 \times 10^{-5}$. It is this fact that explains the large difference between the negligible $\Delta T_{2}$ measured by the WMAP and the one predicted by the inflation hypothesis.

We have to suggest that the COBE measured not the dipole but the contribution of the quadrupole to the Earth's gravitational potential since, besides rather faithful coincidence of the numerical values, there is another surprising agreement.

Quite are army of astrophysicists analyze the WMAP results (we mainly refer to [6-8]) and some teams discovered nearly a complete coincidence in dipole and quadrupole principal axes which fully disagrees with the predicting of the inflation hypothesis.

If we share the opinion of most of the astrophysicists, the COBE measurements of the dipole amplitude $C_{1}$ are directly related to the motion of the Earth about the CMB with $v \approx 390 \pm 60 \mathrm{~km} / \mathrm{sec}$ towards the Lion constellation.
In its turn, this means that a large accumulation of galaxies referred to as the local Superaccumulation moves at a velocity $\sim 600 \mathrm{~km} / \mathrm{sec}$ about the background. It has been believed so far that the centre of this mass must most likely be on rest about the whole distribution of galaxies in the Universe and, hence, about the "relict" background. If the COBE measures the contribution of not the dipole but the quadrupole of the Earth's gravitational field, this treatment no longer arises and the local Superaccumulation is really on rest.

The measurements yielded sensationally small background anisotropy amplitudes with small $\ell: C_{2}, C_{3}, C_{4}$, $C_{5}[2]$

$$
\Delta T_{\ell}(\ell=2,3,4,5) \leq 30 \mu \mathrm{K}
$$

It should be stressed they are small within the frameworks of the inflation hypothesis. And what did the measurements of the Earth's gravitational potential give?

The expression for the $U$ potential in the presence of hydrostatic equilibrium (there is only pressure present and tangential voltages are absent) must contain only even moments $I_{2 n}$ which decrease in magnitude with increasing $n$ :

$$
U=\frac{G M}{R} \cdot\left[1-\sum_{n=2}^{\infty}\left(\frac{R_{e}}{R}\right)^{n} \cdot I_{n} \cdot P_{n}(\cos \theta)+\sum_{n=2}^{\infty} \sum_{m=1}^{n}\left(\frac{R_{e}}{R}\right)^{n} \cdot P_{n}^{m}(\cos \theta)\left(A_{n m} \cos m \lambda+B_{n m} \sin m \lambda\right)\right]
$$

where $A_{n m}, B_{n m}$ are gravitational moments determined by experiments.

The measurements by artificial satellites, however, gave a sensational result, too: all the gravitational moments starting with $I_{3}$ are approximately of the same order $\sim 10^{-6}-10^{-5}$. The decrease of moments with increasing $n$ in this case occurs much slower than has been suspected. This, in its turn, leads to small temperature multifields

$$
\Delta T_{\ell}(\ell=2,3,4,5) \leq 30 \mu \mathrm{K}
$$

which completely agrees with experiment [2] (Figure 2). Note that the ordinate in the diagram is $\Delta T_{1}^{2}$

Figure two shows experimental data without error. For comparison, the results of the first year of WMAP operation are given with measurement errors (Figure 3)

Here we can see again a surprising agreement between the experimental data obtained by COBE and WMAP measurements and the results of harmonic analysis of the gravitational potential:

$$
\begin{array}{ll}
T_{\theta} \sim 2,7 \mathrm{~K} & T_{\mathrm{g}} \sim 2,7 \mathrm{~K} \\
\Delta T_{1} \sim 3 \mathrm{MK} & \Delta T_{2} \sim 3 \mathrm{mK} \\
T_{2} \sim 10 \mu \mathrm{K} & \Delta T_{3} \sim 10 \mu \mathrm{K} \\
\Delta T(\ell=3,4, \cdots, 20) \sim 30 \mu \mathrm{K} & \Delta T(\ell=4, \cdots, 10) \sim 30 \mu \mathrm{K}
\end{array}
$$

The supporters of the inflation hypothesis draw far reaching conclusions observing ups and downs in the angular power spectrum. We cannot produce this diagram since there are restrictions on the harmonic analysis of gravitational multipoles, $\ell \leq 10$, in view of practical needs.

We are not doubted, however, that if gravitational measurements were performed on a large scale and harmonic analysis was made up to $\ell=1000$, we would be bound to produce an oscillating curve for the following reason. As the oblate ness of the Earth $a \sim 1 / 300$ and the gravitational anomalies are very small and a fine anisotropy structure can only be observed just with $\Theta \leq 1^{\circ}$; at large angles the pattern spreads much like in experiments of photon scattering on two slits the diffraction pattern (fine structure) can only be observed when the light wavelength $\lambda$ is smaller than the distance between the slits $d$. If $\lambda<d$, the diffraction pattern disappears.

The geoid heights are proportional to the amplitude of gravitational anomalies. It is rather surprising that the anomalies are not related to the topographic peculiarities of the Earth (mountains, depressions, seas, etc.); the presence of mountains almost does not affect gravimetric measurements. Gravity anomalies are caused by some density fluctuations in the Earth's crust and mantle, are very small in size $\left(a^{2} R \sim 70 \mathrm{~m}\right)$ and so the temperature 


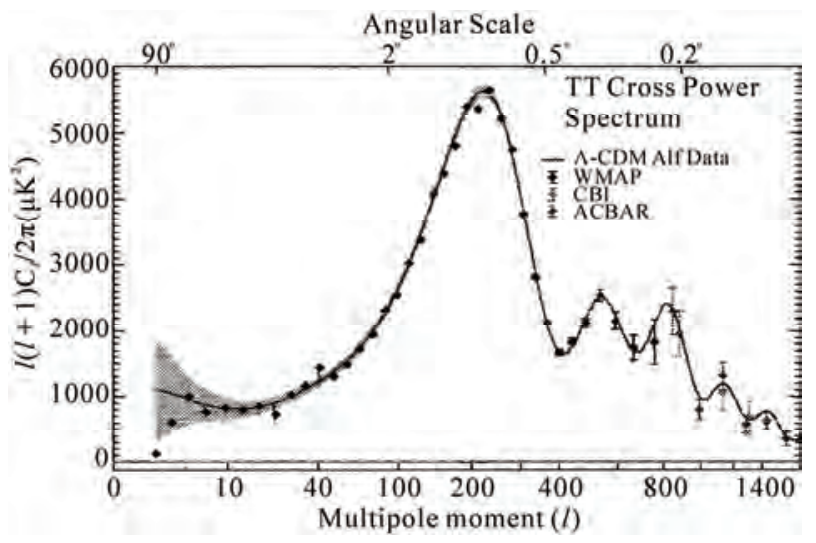

Figure 2. Temperature anisotropy within the frameworks of multipole analysis after the first year of WMAP operation compared with other experiments.

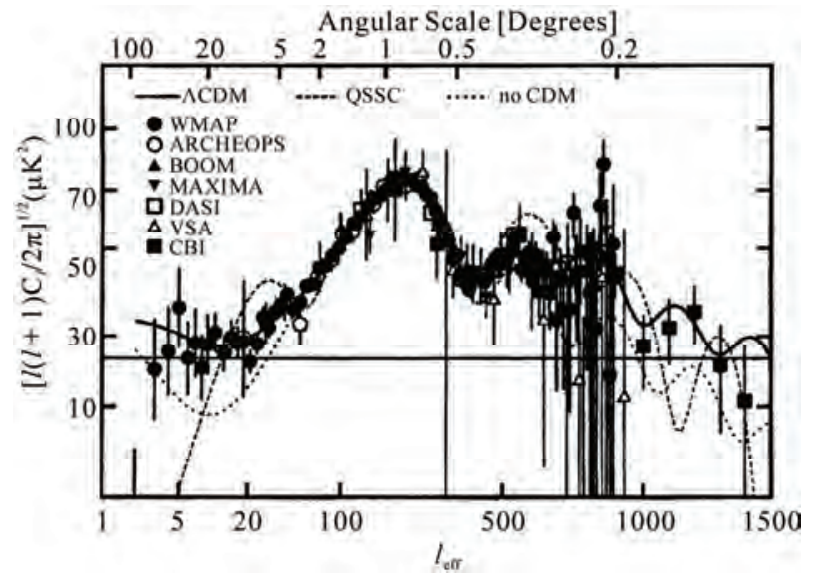

Figure 3. Temperature anisotropy after the first year of WMAP operation.

fluctuations separated by large angles are not related at all.

The above-said is concerned with the $C(\Theta)$ function (Figure 4).

The effect of amplitude smallness with small $\ell$ manifests itself still stronger if we consider the angular correlation function $C(\Theta)$ (Figure 4) instead of spherical function $C_{\ell}$. The $C(\Theta)$ function denotes the correlation degree of temperature fluctuations, an average value for various pairs of dots in the sky separated by an angle $\Theta$.

$$
C(\Theta)=\frac{1}{4 \pi} \Sigma(2 \ell+1) C_{\ell} P_{\ell}(\cos \theta)
$$

The observations [2] show that $C(\Theta)$ is almost equal to zero for angles over $60^{\circ}$; are not related at all.

The absence of wide-angle correlations was first observed by the COBE and now has been confirmed by the WMAP. The smallness of $C(\Theta)$ for wide angles means not only that $C_{2}$ and $C_{3}$ are small but also that the ratio between the first several amplitudes, at least up to $C_{4}$, is

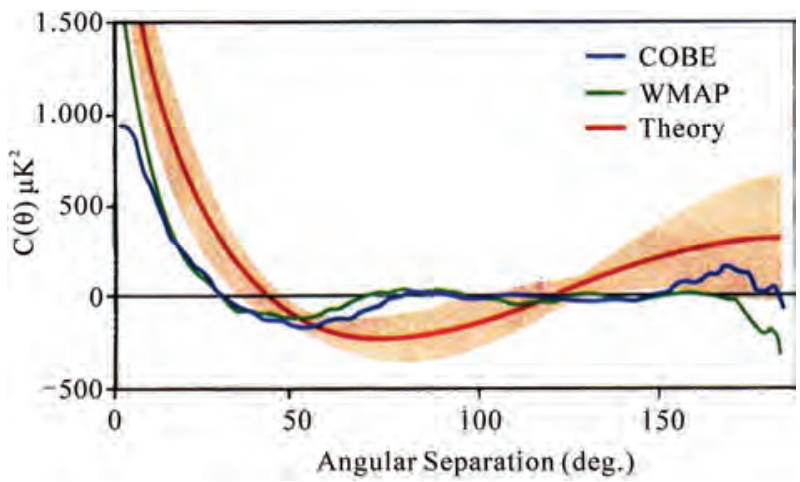

Figure 4. The correlation function of $\mathrm{CMB}$ temperature measured by WMAP and COBE [2].

abnormal too. It should be noted again that a weak power spectrum at wide angles is in a striking contradiction with all the classical inflation models and can be fully explained by the fact that the gravitational anomalies of the Earth are extremely small.

\section{Orientation of Multipoles (Experiment)}

The results of studies into the orientation of many multipoles have become a real sensation bordering mysticism. When dealing with this problem we should refer to [6-8].

1) In 2003 Angelica de Oliveira-Costa and Max Tegmark from Pennsylvania University, Matias Zaldarriuga from Colorado University in Boulder discovered that the main axes of the quadrupole $(\ell=2)$ and octupole $(\ell=3)$ modes are directed closely to each other and have a deficient amplitude. But most of the inflation models suggest that there should not be anything common between these modes.

2) In the same year Hans Christian Eriksen and his colleagues from Norway University in Oslo revealed a coincidence in directions [7]. They divided the sky into various pairs of hemispheres and evaluated the relative amplitude fluctuations on the opposite halves of the sky. The results of their investigation were fully inconsistent with standard inflation cosmology: many pairs of hemispheres differed considerably in power spectrum. But the most unexpected thing is that a pain of the most different hemispheres is evenly divided by an ecliptic, that is, by the plane in which the orbit of motion of the Earth around the Sun lies! (Figure 5).

Besides the too low amplitude with small $\ell$, three more dots can be observed ( $\ell=22, \ell=40$ and $\ell=210$ ) (Figure 2) where the power spectrum considerably differs from the one predicted by inflation models. Despite the fact that these distinctions had been widely known, most of the cosmologists missed the fact that the three deviations correlated with the eclectic, too. 


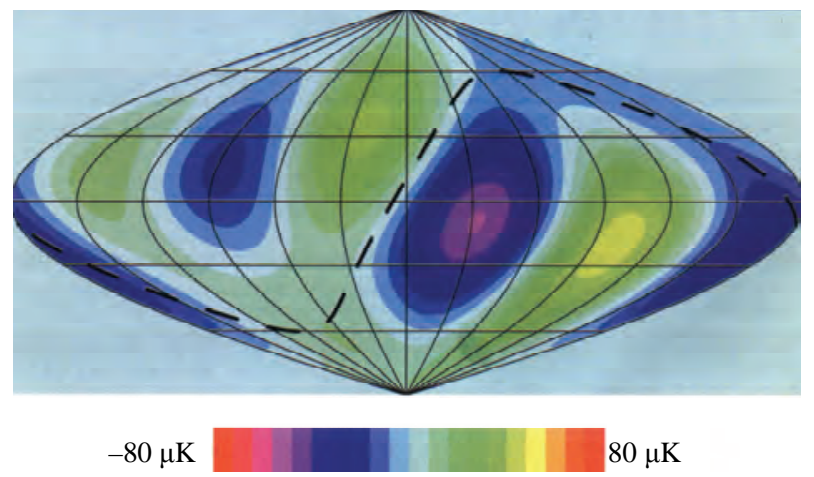

Figure 5. The plane of ecliptic (dashed line) divides the full sky map into a cold and a hot part. The northern part of the ecliptic hemisphere is much colder than the southern one. Account is taken of the contribution of only the quadrupole and the octupole to microwave background anisotropy; the same can be observed in case of higher multipoles as well.

3) In 2004 Domenic J. Schwarz, Glenn Starkman with Craig Copi and Dragan Huterer from the University Cleveland, Ohio, developed a new method of presenting the "relict" background fluctuations in a vector form [8]. This enabled them to verify the speculation that the microwave background fluctuations must not be related to special directions in the Universe.

Also, these scientists discovered unexpected correlations supporting the results obtained by Oliveira Costa and her colleagues [6].

The quadrupole ( $\ell=2$, blue dots -2$)$ and the octupole $(\ell=3$, red dots -1 ) must be random oriented but, instead, they tend to the equinox dots (hollow circles -4 ) and towards the motion of the solar system defined by the dipole axis $(\ell=1$, green dots -3$)$ (Figure 6).

Moreover, their axes lie in the plane of ecliptic (violet line -5$)$. Two of them $(\ell=3$, red dots -1$)$ are in the plane of Supergalaxy, that is, the Local Superaccumulation combining our Galaxy, its adjacent star systems and their accumulations (orange line -6 ). The probability of random coincidence of these directions is $<10^{-4}$ (without regard for the strange properties of low-order multiplets).

The agreement of the dipole and quadrupole axes referred to before and now may have resulted in the situation when the COBE measured the contribution of the gravitational quadrupole (rather than its anisotropy) to the microwave background as it has been mentioned above.

The adherents of "relict" radiation, and they make up the absolute majority, tried to explain the correlation between low modes and the solar system structure by one of the three methods.

First, an error in design of WMAP instruments or wrong analysis of the data obtained (a systematic error). But the WMAP team were rather careful and performed

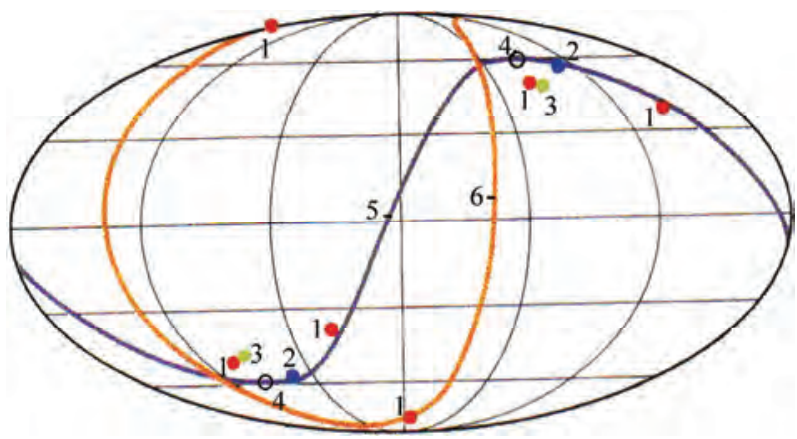

Figure 6. The orientation of the first tree multipoles.

a lot of cross-tests for their instruments. So it is difficult to imagine how the long correlations could arise. Besides, the authors [6-8] disclosed similar correlations in the maps obtained by the COBE that made use of quite different instruments and analysis techniques.

The second explanation shows that there exists an unaccounted source or absorber of microwave photons related to the solar system, for example, an unknown dust cloud on its periphery. But how did this source or absorber of radiation happen to be observed by instruments emitting microwave radiation but was not detected by other numerous astronomical instruments over different wavelength ranges?

At first sight, a discovery of local distortion of microwave background data could solve the problem of its large-scale fluctuations being weak. What actually happens is that it just makes the problem more complicated. With the contribution to radiation associated with hypothetic foreground objects subtracted, the residual cosmological contribution will be much smaller than considered before (any other conclusion would call for a random but exact compensation between the cosmological contribution and the predicted foreground source). In this case it would be much more difficult to assert that the absence of modes with small $\ell$ in the power spectrum is just freak of chance.

And, finally, the third attempt to explain only the absence of modes with small $\ell$ in the power spectrum is connected with topology since it has been impossible so far to invent a physical mechanism for their suppression. It is evident that these attempts cannot satisfy us because they are ad hoc hypotheses in the proper sense of this word.

\section{Orientation of Gravitational Multipoles}

To understand all the surprises connected with the microwave background we should again consider such a fundamental physical phenomenon as the gravitational energy levels formed by the Earth.

As it has already been mentioned several times, the 
shape of the Earth just slightly differs from a sphere, its oblate ness $a \sim 1 / 300$, and the South pole is only $30 \mathrm{~m}$ closer to the centre of the Earth than the North pole.

When expanding a gravitational potential in terms of Legandre polynoms we only apply one spherical coordinate system $(R, \Theta, \lambda)$ and, hence, the directions of the principle axes of a quadrupole, octupole and other higher multipoles theoretically agree, and the Earth's symmetrical and equilibrium shape "confirms" this agreement.

One of the significant discoveries of the present time is spotting extremely intense radiation at distances of up to several Earth's radii [5]. The intensity of this radiation is millions of times higher than that of the cosmic rays observed until recently in the terrestrial atmosphere. Later on, the zones, where particles captured by the geomagnetic field are concentrated, were given the name radiation belts. Low-energy electrons fill almost the whole magnetic sphere of the Earth. The outer boundary of the magnetic sphere is at a distance $r \geq 10 R_{\oplus}$. A little the studies into radiation detected a new physical phenomenon predicted by E. Parker: an ionized gas flow, referred to as the solar wind, travels from the Sun at a velocity $\sim 400-600 \mathrm{~km} / \mathrm{sec}$ and replenishes steadily the number of charged particles in the Earth atmosphere. At calm periods the intensity of electrons may be as high as $J \sim 10^{8} \mathrm{~cm}^{-2} \cdot \mathrm{sec}^{-1}$ and their spatial concentration varies between several particles and several tens per $1 \mathrm{~cm}^{3}$. During magnetic radiations their variation comes to two orders. The inner part of the magnetic sphere lying a dipole-like geomagnetic field (up to $3 R_{\oplus}$ ) is referred to as the plasma sphere. The concentration of "cold plasma” particles in the plasma sphere is $\sim 10^{4} \mathrm{~cm}^{-}$.

Hence, around the Earth there is a sufficient quantity of electrons whose transition from one gravitational energy level onto a lower-lying level is followed by emission of low-energy photons forming quasi-black-bodied radiation - CMB. The number of microwave background photons in a unit volume must be $n \sim 410$.

The electric charge of the Earth in this case does not increase since the emitted electrons pass again onto higher-lying levels (the Earth's temperature is 280 K) and the whole process of radiation reminds a process with any black body. The only difference is that the levels are gravitational now rather than Coulomb-like.

Since the Sun is the only main supplier of electrons onto the Earth, the CMB spectrum reflects this dependence, an ecliptic dependence.

Another source of particles may be the ionized shell round the Sun where the electron energy comes to just several electron-volts.

Electron trapping has an interesting feature: the field of trapping differs on the night and the day sides of the
Earth. On the day side it spreads almost to the boundaries of the magnetic sphere whereas on the night side it takes just a small part of the latter.

This effect manifests itself particularly strongly at the moment when the Earth is at the equitoctial points, that is, when the day is equal to the night. That is why the dependence of the CMB spectrum on the equitoctial points is so mysterious.

It should be said finally how the two octupole axes $(\ell=$ 3) have been brought into the Supergalaxy plane. It is well known that the velocity of the Galaxy about the Supergalaxy is estimated at 400-500 km/sec. But, strange as it may seem, this velocity is directed at an angle $\approx$ $120^{\circ}$ to, as it is widely believed, the direction of the Earth's motion relative to the CMB determined by the dipole axis. But, as it has been mentioned, the dipole axes almost coincide with the quadrupole and the octupole axes (Figure 6). The angle between the octupole axes, in its turn, is $60^{\circ}$. So it is not surprising that a pair of octupole axes lies in the Supergalaxy plane.

\section{Microwave Background Polarization}

It is well known that the Earth has a magnetic field. The geomagnetic field induction $\boldsymbol{B}$ differs in magnitude and direction at different points of the Earth's surface. Besides, the geomagnetic field elements remain constant in time but all the time change their values.

The fact that the Earth has a magnetic field is responsible for partial polarization of such elementary particles as electrons, protons and neutron near the planet. This polarization depends on the place and the time of observation, that is, it fluctuates strongly. When passing from one gravitational level onto another one electrons and protons emit photons which get partially polarized thanks to their parents, and this polarization will fluctuate, too.

The solar plasma (solar wind) colliding with the magnetic sphere of the Earth begin to flow around it. The positive particles in this case are deflected eastward and the negative particles westward. The flow is subjected to polarization which can be described by an equivalent current $\boldsymbol{J}$ counter-clockwise directed as viewed from the North Pole. The new WMAP information [9] gives stunning verification of this surprising polarization directivity of the photons emitted by polarized electrons (Figure 7) partial polarization to the "relict" radiation generated during recombination is in doubt since over the period elapsed, $13 \times 10^{9}$ years, this partial polarization should have died away inevitably because of multiple elastic scattering.

In a first approximation the geomagnetic field is presented by a magnetic dipole at $\sim 340 \mathrm{~km}$ from the centre of the Earth. The investigations performed by Earth 


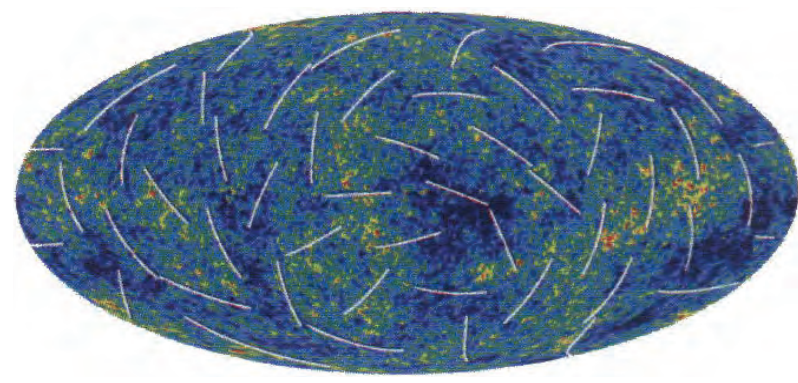

Figure 7. The white dashes denote the direction of microwave background polarization.

satellites have shown that a field may be considered dipole only to a rough approximation [10]. The inclusion of the quadrupole and the octupole (except the dipole) expansion terms of the real earth field by spherical harmonics gives a much better agreement with experiment.

In $1838 \mathrm{~K}$. Gauss developed a general theory of analytical representation of the geomagnetic field as functions of the position of points on the Earth's surface. In the spherical coordinate system $(r, \Theta, \lambda)$ Gauss obtained the following expression for magnetic potential

$$
U=\sum_{n=1}^{\infty} \sum_{m=0}^{n} \frac{R_{\oplus}^{n+1}}{r^{n+1}}\left(g_{n}^{m} \cos m \lambda+h_{n}^{m} \sin m \lambda\right) P_{n}^{m}(\cos \theta)
$$

where $R_{\oplus}$ is the earth's radius, $g_{m}^{n}$ and $h_{m}^{n}$ are constant coefficients determined experimentally. It can be seen that in studying microwave background polarization the expansion in terms of spherical harmonics can be naturally substantiated as opposed to the inflation hypothesis.

The mean geomagnetic induction of the Earth is $\sim 5 \times$ $10^{-5} \mathrm{~T}$. Then the energy of interaction of an electron with the geomagnetic field will be

$$
E=\mu_{e} \cdot B \approx 3 \times 10^{-9} \mathrm{eV} .
$$

This energy corresponds to the contribution to the CMB temperature

$$
\Delta T_{4}^{2} \approx(10.8 \pm 2.4) \mu \mathrm{K}^{2}
$$

According to [10] he geomagnetic field is the sun of three fields:

1) basic magnetic field, 95\%;

2) abnormal geomagnetic field, 4\%;

3) electromagnetic external field, $\sim 1 \%$.

The spherical harmonic analyses show that the basic geometric field consists of a dipole fraction (over $80 \%$ ) and a non-dipole one. The octupole contribution $(\ell=3)$ is below $10 \%$. The contribution of the next multipole $(\ell=$ 4) to CMB anisotropy will be, all the more, less than $10 \%$

$$
\Delta T_{4} \leq 3 \mu \mathrm{K}
$$

or

$$
\Delta T_{4}^{2} \approx 10 \mu \mathrm{K}^{2}
$$

According to the WMAP data [2], the contribution of the multipole $(\ell=4)$ to CMB anisotropy (Figure 8) is

$$
\Delta T_{4}^{2} \approx(10.8 \pm 2.4) \mu \mathrm{K}^{2}
$$

(The data of the diagram should be multiplied by $\ell$ )

Now we have to do nothing but to note another surprising agreement of data (16) and (17).

All the calculations of the geomagnetic field were performed with a restriction on the eighth-order terms $n \leq 8$. Therefore, as with the gravitational field, we cannot study the ups and downs on the oscillating curve of CMB polarization power spectrum.

An important feature of the dipole magnetic field of the Earth is a high degree of uniformity in small volumes, its gradients with respect to the radius and the meridian do not exceed $(10-20) \times 10^{-3} \mathrm{~T} / \mathrm{km}$. So the oscillation of the curve (Figure 7) does not surprise us, were neither ups nor downs at all. And since the magnetic anomalies of the Earth are slight, oscillations may show up only at small angles of CMB anisotropy observation $\Theta \leq 1^{\circ}$.

The detection of negative magnetic anomalies $\boldsymbol{B}_{\boldsymbol{a}}$ has been a fundamental discovery. Their number is found to be comparable with positive $\boldsymbol{B}_{\boldsymbol{n}}$. An anomaly is called negative if its field in the northern hemisphere is directed to the upper part of space over a horizontal plane so that $\boldsymbol{B}_{\boldsymbol{a}}$ is nearly parallel with $\boldsymbol{B}_{\boldsymbol{n}}$. The experimental WMAP data (Figure 7) perfectly illustrates this peculiarity of the geomagnetic field.

The systematic measurements of the elements or the module of the geomagnetic field at different points of the Globe have shown that the geomagnetic field has a complicated distribution over the Earth's surface and the magnetic field of the northern hemisphere differs essentially from that of the southern one.

Since the magnetic field governs the flow of electrons falling on the Earth, the intensity of the photons emitted by the electrons will differ in the hemispheres. This is an

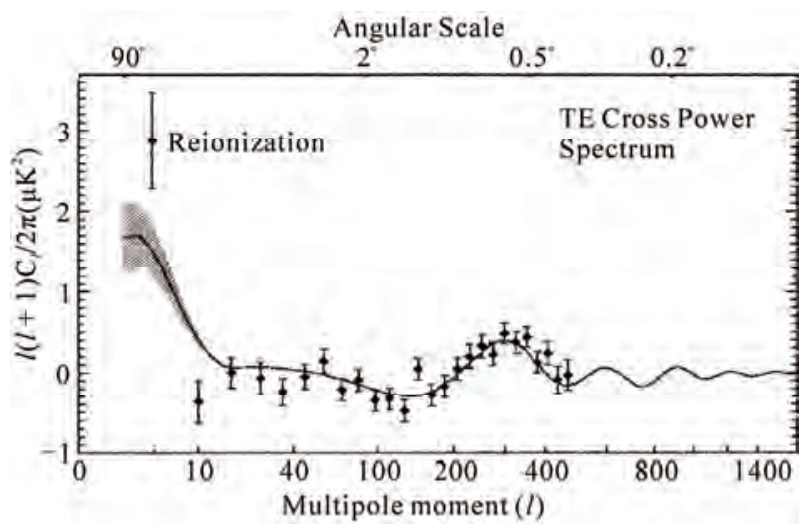

Figure 8. The power spectrum of temperature polarization. 
explanation of the effect discovered by Hans Ericsen and his colleagues: a pair of the most varying hemispheres with respect to power spectrum is exactly divided by the ecliptic.

The authors [2] place particular emphasis upon the fact that each mode of the measured CMB anisotropy exhibits a Gaussian distribution with nearly the same width $\Gamma$. This property of anisotropy can be naturally explained if we consider the radiation spectrum of atoms.

From its excited state an atom spontaneously passes into a lower energy state. The lifetime of excited atomic states $\tau$ has a definite value and varies between $10^{-8}$ and $10^{-9} \mathrm{sec}$ for different atoms. The possibility of spontaneous transitions shows that excited states cannot be considered strictly stationary. In this connection the energy of excited states is not exactly definite and an excited energy level has a width $\Gamma$. Owing to the finite width of excited levels the energy of the photons emitted by the atoms has a spread described by a curve referred to as a Gaussian.

Since any energy levels are eventually determined by the spatial structure deformation, one should expert that the gravitational energy levels will have a finite width, too, and the energy spread will be described by a Gaussian. The width $\Gamma$ of all the levels in this case will be almost the same because we have got only one gravitational "atom" - the Earth.

Gravitational energy levels are no worth than $\mathrm{Cou}-$ lombian ones and, hence, each mode of CMB anisotropy (difference) must have a Gaussian distribution with the same width which agrees with the WMAP data.

And finally the last, not so important though, remark on the polarization power spectrum.

The gravitational and the geomagnetic fields of the Earth have quite different structures; besides, the geomagnetic poles differ slightly from the geographic poles. It is because of this fact that the ups and the downs in the diagrams of angular and polarization power spectra do not agree (Figure 1 and Figure 7).

\section{Conclusions}

Our time is realistically called the golden age of astrophysics as wonderful and, most often, unexpected discoveries in the world of stars follow one after another now.

In 1929 Einstein said in one of his speeches: "Frankly speaking, we want not only to learn how nature is arranged but also to accomplish as possible an Utopian and seemingly bold aim - to understand why nature is just as it is. This is a Prometheus element of scientific work".

The whole material of this article, both numerical and topological, testifies that in 1965 Penzias and Wilson discovered not "relict" radiation but a new fundamental phenomenon: any body in the Universe forms around itself gravitational energy levels which, among other things, are responsible for the quasi-black-bodied radiation of the Earth with $T \sim 2.7 \mathrm{~K}$.

This prediction fully agrees with the principle of observables: in science there must not be any concepts which cannot be formulated in the language of real or mental experiments.

By irony of fate, energy levels were first discovered not in space by the naked eye (planet orbits) but in microcosm as a result of titanic efforts of experimenters and great discoveries of theorists.

Quantization has been so far a privilege of microcosm, and the discovery of gravitational energy levels formed by macrobodies deprives it of this privilege demonstrating us the fact of quantization of the whole Universe. The difference between microcosm and macrocosm is only that, when making a Gobelin tapestry of macrocosm, nature uses longer threads.

In his traditional lecture at the Nobel Prize ceremony R. Wilson recalled that in 1965 in their first report on their results the authors tried to avoid discussing the cosmological explanation of their discovery. "We believe that our results do not depend on their theoretical interpretation and can survive any of them". Our microwave background analysis has shown that their prudence was not unnecessary.

If the WMAP team had envisaged from the very beginning measuring not the difference in microwave background flows (anisotropy) $\Delta i$ but had measured, at least for one wave, the absolute flow $i$ far away from the Earth, the problem of CMB physical nature would have been solved.

The absolute flow of CMB $i \sim 1 / \mathrm{R}^{2}$ at the distance of $1.5 \cdot 10^{6} \mathrm{~km}$ (the zone of WMAR action) must be 55311 times less than the one measured near the Earth if this background was formed due to the gravitation level of the Earth. In this case the temperature in the zone of WMAP action must come to

$$
\langle T\rangle \approx 2.725 / 55311 \approx 49 \times 10^{-6} \mathrm{~K}
$$

Figure 2 enables us to calculate the mean value of anisotropy

$$
\Delta T \approx 45 \times 10^{-6} \mathrm{~K} .
$$

The natural question arises: does this magnitude $\Delta T$ account for the absolute flow I, i.e. the value of the temperature T measured by the WMAP? If it is true, the WMAP has discovered the effect of gravitation energy levels and closed "relict" radiation.

To solve this dilemma to the end we must perform a simple and cheap experiment ("experimentum crucis"). 
For this purpose, of the sattelites launched to geostationary orbit ( $36000 \mathrm{~km}$ from the earth) must have a bolometer aboard which is to measure the absolute flow $\mathrm{CMB}$ in the microwave range just for one wavelength. If the intensity I interns out to be equal to that measured on the Earth, the CMB will be "relict". Otherwise the effect of gravitational energy levels will be confirmed.

A similar situation took place in 1955 when Lee and Yang proposed that parity is not conserved in weak interactions. There were not, however, any experiments performed that would support their assumption. Soon Mrs. Wu succeeded in performing such an experiment and, as a result, Lee and Yang were awarded a Nobel prize. This experiment will solve the fate of graviton.

\section{References}

[1] A. A. Penzias and A. R. W. Wilson, "Early cosmic background,” Astrophysics Tournament, Vol. 142, 1965, pp. 419-425.

[2] G. L. Bennet, et al., "First Year Wilkinson Microwave
Anisotropy Probe \{WMAP\} Observation,” Astrophysics Journal, Vol. 148, 2003, pp. 1-28.

[3] R. A. Alpher and R. C. Herman, "Evolution of the Universe,” Nature, Vol. 162, 1948, pp. 774-780.

[4] K. Huang, "Statistical mecanics," John Wiley Sons, Inc., New York, 1963.

[5] V. S. Murzin, "Physics of Cosmic Ray," Moscow State Universitety, Moscow, 1970.

[6] A. de Oliveira-Costa, et al., "Cosmik Microwave Background Anisotropies in Multiconnected Flat Spaces," Physics Review, Vol. D69, 2004, 103518.

[7] N. K. Eriksen, et al., "Asymmetries in the Cosmic Microwave Background Anisotropy Field,” Astrophysics Journal, Vol. 605, No. 14, 2004.

[8] D. J. Schwarz, et al., "Large-Angle Anomalies in the CMB,” arXiv:astro-ph/0403353.

[9] M. Turler, "New WMAP Results Give Support to Inflation,” CERN Courier, Vol. 46, No. 4, 2006, p. 12.

[10] V. A. Magnitsky, “General Geophysics,” Moscow State Universitety, Moscow, 1995. 